\title{
OPEN Ecological adaptation and phylogenetic analysis of microsymbionts nodulating Polhillia, Wiborgia and Wiborgiella species in the Cape fynbos, South Africa
}

Tiisetso Mpai ${ }^{1}$, Sanjay K. Jaiswal ${ }^{3 \bowtie}$, Christopher N. Cupido ${ }^{2}$ \& Felix D. Dakora ${ }^{3 \bowtie}$

Polhillia, Wiborgia and Wiborgiella species are shrub legumes endemic to the Cape fynbos of South Africa. They have the ability to fix atmospheric $\mathrm{N}_{2}$ when in symbiosis with soil bacteria called 'rhizobia'. The aim of this study was to assess the morpho-physiological and phylogenetic characteristics of rhizobia associated with the nodulation of Polhillia, Wiborgia and Wiborgiella species growing in the Cape fynbos. The bacterial isolates from root nodules consisted of a mixture of fast and intermediate growers that differed in colony shape and size. The isolates exhibited tolerance to salinity $(0.5-3 \% \mathrm{NaCl})$ and $\mathrm{pH}(\mathrm{pH} \mathrm{5-10)}$ and different antibiotic concentrations, and could produce 0.51 to $51.23 \mu \mathrm{g} \mathrm{mL}^{-1}$ of indole-3-acetic acid (IAA), as well as solubilize tri-calcium phosphate. The ERIC-PCR results showed high genomic diversity in the rhizobial population and grouped them into two major clusters. Phylogenetic analysis based on 16S rRNA, atpD, glnll, gyrB, nifH and nodC gene sequences revealed distinct and novel evolutionary lineages related to the genus Rhizobium and Mesorhizobium, with some of them being very close to Mesorhizobium australicum. However, the phylogenetic analysis of $\mathrm{glnll}$ and nifH genes of some isolates showed incongruency.

Polhillia, Wiborgia and Wiborgiella species belong to the family Leguminosae and tribes Genisteae and Crotalarieae $^{1-3}$. They are endemic to the Cape fynbos biome, recognized as one of the richest areas of flowering plants in the world ${ }^{4-6}$. These legumes have bright yellow and/or white flowers, which are a major attraction for tourists 3,7 . They also contribute to the fertility of fynbos soil through $\mathrm{N}_{2}$ fixation with native soil rhizobia. In fact, Polhillia brevicalyx, Polhillia pallens, Wiborgia sericea, Wiborgia tetraptera, Wiborgia obcordata and Wiborgiella sessilifolia are reported to derive between 61 and $91 \%$ of their $\mathrm{N}$ nutrition from symbiotic $\mathrm{N}_{2}$ fixation ${ }^{8}$.

Some rhizobial bacteria are capable of tolerating acidic conditions, often characterised by high $\mathrm{H}^{+}$concentration and the increased solubility of heavy metals and trace elements ${ }^{9,10}$, as well as tolerance to high soil salinity which can inhibit bacterial survival, growth and persistence ${ }^{11}$. Some bacteria can also solubilize P from unavailable soil $\mathrm{P}$ complexes for plant uptake as well as produce IAA, a hormone that is involved in root formation and root elongation for increased uptake of water and nutrients $s^{12,13}$. The identification of acid, salinity and antibiotic tolerant rhizobia with the ability to produce IAA and solubilize $\mathrm{P}$ is a first step to selecting rhizobia for inoculant production.

Rhizobia nodulating various Cape fynbos shrub legumes have been reported ${ }^{14-17}$. However, information on the microsymbionts nodulating Polhillia, Wiborgia and Wiborgiella species endemic to the Cape is lacking. Therefore, the aim of this study was to evaluate the morpho-physiological diversity and phylogeny of bacterial symbionts associated with the nodulation of Polhillia, Wiborgia and Wiborgiella species. We hypothesized that the rhizobial strains nodulating these legumes have genomic stability and were same type of rhizobial species

${ }^{1}$ Department of Crop Sciences, Tshwane University of Technology, Private Bag X680, Pretoria 0001, South Africa. ${ }^{2}$ Department of Botany, University of Fort Hare, Alice, South Africa. ${ }^{3}$ Department of Chemistry, Tshwane University of Technology, Arcadia Campus, Private Bag X680, Pretoria 0001, South Africa. ${ }^{\circledR}$ email: sanjaysiswa@ gmail.com; Dakorafd@tut.ac.za 


\begin{tabular}{|c|c|c|c|c|c|c|}
\hline \multirow[b]{3}{*}{ Species } & \multirow[b]{3}{*}{ Sample site } & \multirow[b]{3}{*}{ Geographic co-ordinates } & \multirow[b]{3}{*}{$\mathrm{pH}\left(\mathrm{H}_{2} \mathbf{0}\right)$} & \multicolumn{3}{|c|}{ Soil chemical properties } \\
\hline & & & & $\mathrm{NH}_{4}^{+}$ & $\mathbf{P}$ & $\mathrm{Zn}$ \\
\hline & & & & \multicolumn{3}{|l|}{ mg.kg ${ }^{-1}$} \\
\hline Polhillia pallens & Witkoppies farm & $\begin{array}{l}34^{\circ} 33^{\prime} 53^{\prime \prime} \mathrm{S} \\
19^{\circ} 59^{\prime} 43^{\prime \prime} \mathrm{E}\end{array}$ & $4.45 \pm 0.05$ & $0.60 \pm 0.00$ & $43.50 \pm 0.50$ & $2.84 \pm 0.17$ \\
\hline Wiborgia obcordata & Bushmans Kloof & $\begin{array}{l}32^{\circ} 07^{\prime} 14^{\prime \prime} \mathrm{S} \\
19^{\circ} 06^{\prime} 28^{\prime \prime} \mathrm{E}\end{array}$ & $4.33 \pm 0.09$ & $0.04 \pm 0.01$ & $6.00 \pm 0.58$ & $0.18 \pm 0.01$ \\
\hline Wiborgia sericea & Travellers Rest farm & $\begin{array}{l}32^{\circ} 04^{\prime} 15^{\prime \prime} \mathrm{S} \\
19^{\circ} 04^{\prime} 32^{\prime \prime} \mathrm{E}\end{array}$ & $4.47 \pm 0.09$ & $0.03 \pm 0.00$ & $8.33 \pm 0.33$ & $0.66 \pm 0.03$ \\
\hline Wiborgiella sessilifolia & Bredasdorp/Elim Pass & $\begin{array}{l}34^{\circ} 37^{\prime} 58^{\prime \prime} \mathrm{S} \\
19^{\circ} 49^{\prime} 399^{\prime \prime} \mathrm{E}\end{array}$ & $8.20 \pm 0.00$ & $0.67 \pm 0.48$ & $15.67 \pm 0.33$ & $0.41 \pm 0.01$ \\
\hline
\end{tabular}

Table 1. Summary of plant species, sample sites and soil chemical properties.

due to the restricted habitat of these wild fynbos legumes. To test these hypotheses, the following questions were addressed (1) Which rhizobial species nodulate these wild shrub legumes? (2) What are the phylogenetic behaviours of the isolates?

\section{Materials and methods}

Nodule sampling and description of study sites. Root nodules were collected from Wiborgiella sessilifolia and Wiborgia sericea at Bredasdorp and Travellers Rest farm, respectively (Table 1). Due to the limited number of Polhillia pallens plants in the Witkoppies farm, as well as the difficulties in uprooting Wiborgia obcordata plants in their natural stands, mature seeds and rhizosphere soil samples were collected from their respective sites (Table 1) and used to trap rhizobia in the glasshouse. All the methods were performed in accordance with the relevant regulations and guidelines. Collecting root nodules, seeds and rhizosphere soil samples was done randomly according to plant availability at each site during the wet (July to September 2018) season.

Trapping rhizobia and their isolation. Seeds of $P$. pallens and $W$. obcordata were pre-germinated using the acid scarification method ${ }^{18,19}$ and transplanted into sterile sand in pots ${ }^{20}$. Seedlings were inoculated with their respective rhizosphere soil suspension ${ }^{21}$. Five replicate pots were used for each treatment. All seedlings were supplied with $\mathrm{N}$-free fahraeus solution as a source of nutrients ${ }^{22}$. After 42 days of growth in the glasshouse, effective root nodules with a red or pinkish colour were harvested from the glasshouse-grown plants for rhizobial isolation. The root nodules obtained from the field and those from the glasshouse were surface sterilized and used to isolate rhizobia, following standard procedures ${ }^{20}$.

Rhizobial authentication. Surface sterilised pre-germinated seeds of $P$. pallens, and W. sessilifolia, were transplanted in sterilized plastic pots $\left(1.2 \mathrm{dm}^{3}\right)$ containing autoclaved sand. Each seedling was inoculated with $1 \mathrm{~mL}\left(10^{7}\right.$ to $10^{8}$ rhizobial cells $\left.\mathrm{ml}^{-1}\right)$ of the test bacterial culture under axenic conditions. The pots with seedlings were then transported to the glasshouse and left to grow under glasshouse conditions. Three replicate pots were used for each isolate and the plants were watered twice a week with $\mathrm{N}$-free ferrous nutrient solution ${ }^{22}$. Similarly, all the test isolates were used to also inoculate cowpea seedlings in a host-range test.

DNA extraction and ERIC-PCR genomic fingerprinting. Genomic DNA of the rhizobial isolates grown in YMB $\left(\sim 1 \times 10^{9}\right.$ rhizobia cells $\left.\mathrm{ml}^{-1}\right)$ was extracted using a GenElute Bacterial Genomic DNA kit, according to the manufacturer's instructions (Sigma Aldrich, USA).

Fingerprints of the rhizobial isolates were evaluated by the ERIC-PCR method performed in a $15 \mu \mathrm{l}$ reaction mixture containing $1 \mu \mathrm{l}$ DNA (50-80 ng $\left.\mu^{-1}\right), 7 \mu \mathrm{l} 2 \times$ My Taq PCR master mix, $1 \mu \mathrm{l}$ each ERIC forward and reverse primers and $5 \mu$ d double distilled sterilised water.

Amplification was performed in a Thermal cycle (T100 BIORAD, USA). The primers used and the amplification conditions are shown in Table S1. The PCR-amplified products were analysed by horizontal gel electrophoresis at $100 \mathrm{~V}$ for $3.0 \mathrm{~h}$ in a $1.2 \%$ agarose gel stained with ethidium bromide $\left(1 \mu \mathrm{g} \mathrm{ml}^{-1}\right)$ in $1 \mathrm{X}$ TAE buffer. A standard molecular marker (GeneDirex $1 \mathrm{~kb}$ ladder) was included to estimate the size of the fragments. The gels were photographed under UV illumination using a gel documentation system (Geldoc ${ }^{\mathrm{Tm}} \mathrm{XR}+$, Bio-RAD, USA).

Cluster analysis. The banding patterns were scored directly from the gel photographs and the isolates were grouped through visual inspection of the banding. The DNA fingerprints (bands) obtained from the ERIC-PCR products was used for cluster analysis. Only distinct, well-resolved, and unambiguous bands were scored faint bands and $\leq 50 \mathrm{bp}$ band sizes were excluded in the cluster analysis. A binary scoring system ( 1 for presence and 0 for the absence of a band) was used to generate an input matrix. This was analyzed by means of the Unweighted Paired-Group Method with arithmetic mean (UPGMA) ${ }^{23}$. A dendrogram was then generated from the matrix using NTSYS-Pc software ${ }^{24}$.

Amplification of the 16S rRNA, housekeeping and symbiotic genes. The genomic DNA of representative rhizobial strains of different clusters in the ERIC-PCR dendrogram were amplified with primers for 16S rRNA, housekeeping ( $a t p D, g y r B$ and $g l n I I)$ and symbiotic ( $n i f H$ and nodC) genes. Amplification was performed 
in a $25 \mu \mathrm{l}$ reaction mixture containing $1 \mu \mathrm{l}(50-80 \mathrm{ng})$ of genomic DNA template, $3 \mu \mathrm{l} 5 \times$ My Taq Buffer, $1 \mu \mathrm{l}$ $(10 \mu \mathrm{M})$ forward primer, $1 \mu \mathrm{l}(10 \mu \mathrm{M})$ reverse primer, $0.1 \mu \mathrm{l}(5 \mathrm{U})$ Taq polymerase (Bioline, USA) and $18.9 \mu \mathrm{l}$ sterile distilled water. Amplifications were performed in a Thermal cycle (T100 BIORAD, USA). The primers used, and amplification conditions are indicated in Table S1. The amplified products were separated by electrophoresis at $80 \mathrm{~V}$ for $1 \mathrm{~h}$ in a $1.2 \%$ agarose gel stained with ethidium bromide $\left(1 \mu \mathrm{g} \mathrm{ml}^{-1}\right)$ in $1 \mathrm{X}$ TAE buffer. Standard molecular markers (GeneDirex $100 \mathrm{bp}$ and $1 \mathrm{~kb}$ ladders) were included to estimate the length of the fragments.

Sequencing of the $16 \mathrm{~S}$ rRNA, atpD, gyrB, glnll, nifH and nod $C$ genes and their processing. The amplified PCR products were purified using PCR clean up kit (NEB, USA) and sent to Macrogen company, The Netherlands, for sequencing. The quality of the sequences were assessed using BioEdit 7.0.0 software ${ }^{25}$. Closely related species were identified using the BLAST $\mathrm{n}$ (Basic Local Alignment Search Tool) program in the NCBI (National Centre for Biotechnology Information) server. The 16S rRNA, atpD, gyrB and $\operatorname{glnII}$, nodC, and nifH gene sequences of the reference or type strains used in this study were retrieved from the NCBI-GenBank database. Close reference type strain sequences from the NCBI GenBank database were selected and aligned with sequences of the test strains using MUSCLE ${ }^{26}$, and used to construct phylogenetic trees using the MEGA 6.0 program $^{27}$. Phylogenetic trees were generated using the P-distance method to calculate evolutionary distance ${ }^{28}$, and evolutional history was inferred using the Maximum likelihood metho ${ }^{29}$ algorithm with 1000 bootstraps to allow for a strong support ${ }^{30}$. The MEGA 6 program was used to calculate transition-transversion-ratio to know the content of homoplasy.

Biochemical and physiological characterization of isolates. The rhizobial isolates were grown in YMB which was adjusted to different $\mathrm{pH}$ levels $(\mathrm{pH} 3,5,9$ and 10). The YMB media at $\mathrm{pH} 7$ was used as a control. To screen for $\mathrm{pH}$ tolerance, $10 \mu \mathrm{L}\left(\approx 10^{8}\right.$ cells $\left./ \mathrm{mL}\right)$ of freshly prepared broth culture of each isolate was dropped into $4 \mathrm{~mL}$ of freshly made broth previously adjusted to the different $\mathrm{pH}$ levels ${ }^{20}$. Thereafter, they were incubated at $28^{\circ} \mathrm{C}$ for seven days with constant agitation $(200 \mathrm{rpm})$ on a shaker. The $\mathrm{pH}$ levels of 5 and 6 were maintained with a buffer using $40 \mathrm{mM}$ MES, while $30 \mathrm{mM}$ HEPES was used for $\mathrm{pH} 7$ and 9 , and $30 \mathrm{mM}$ CHES for $\mathrm{pH} 10^{31,32}$. After seven days of incubation, the optical density of the broth cultures was measured at $660 \mathrm{~nm}$ using vis spectrophotometer (7300 Jenway UK).

The phosphate solubilization test was done using double agar layer plates containing B3 media (basal layer) and tri-calcium phosphate $\left[\mathrm{Ca}_{3}(\mathrm{PO} 4)_{2}\right]$ (top layer), as described by Dabo et al. ${ }^{33}$. The diameter of the halo zone produced around each bacterial colony was measured and taken as indicative of P-solubilizing activity. The phosphate-solubilizing index (PSI) of each isolate was derived as the ratio of the diameter of the halo zone (R) and colony diameter $(\mathrm{r})$.

A colorimetric method was used to test for IAA production by isolates in tryptophan-supplemented YMA broth, as described by Ibny et al. ${ }^{13}$.

To test for salt $(\mathrm{NaCl})$ tolerance of the rhizobial isolates, a $20 \mu \mathrm{l}$ volume of each matured bacterial isolate was dropped on a YMA plate containing different concentrations $(0.5 \%, 1 \%, 2 \%$ and $3 \%)$ of $\mathrm{NaCl}$, with $0.01 \% \mathrm{NaCl}$ as the control ${ }^{13}$.

Intrinsic antibiotic resistance. Rhizobial growth was tested in YM agar media supplemented with different concentrations of each antibiotic: streptomycin $\left(50,100\right.$, and $\left.200 \mu \mathrm{g} \mathrm{ml}^{-1}\right)$, kanamycin, chloramphenicol and ampicillin $\left(25,50\right.$ and $\left.75 \mu \mathrm{g} \mathrm{ml}^{-1}\right)$ as well as neomycin $\left(1,5\right.$ and $\left.10 \mu \mathrm{g} \mathrm{ml}^{-1}\right)$ with $0 \mu \mathrm{g} \mathrm{ml} \mathrm{m}^{-1}$ antibiotic as a control ${ }^{34}$. All assays were done in triplicates. Colony growth was assessed after incubation at $28^{\circ} \mathrm{C}$. Isolates showing growth in all triplicate plates were considered tolerant, and isolates which did not grow, were considered susceptible to that antibiotic concentration.

\section{Results}

Rhizobia isolated. The original host plants (Polhillia pallens and Wiborgia obcordata) were able to nodulate with rhizosphere soil suspensions from their respective sites of collection. (Table S2). After isolation, a total of 35 isolates were obtained, five obtained from Wiborgiella sessilifolia, ten from Polhillia pallens, five from Wiborgia sericea, and 15 from Wiborgia obcordata, (Table S2).

Morpho-physiological characterization of rhizobial isolates. About $36 \%$ of the isolates were fastgrowers which took 2 to 4 days to appear on yeast mannitol agar (YMA) plates, while the remaining isolates exhibited intermediate growth rate (Table S2). Furthermore, $94 \%$ of the isolates showed small colony size ( $\leq 1-2 \mathrm{~mm}$ diameter), $77 \%$ were non-elastic in texture and cream white in colour, while $83 \%$ showed a flatround shape.

Authentication and host range test of rhizobial isolates. The 35 test isolates were tested for host range under glasshouse conditions. Two isolates from Wiborgiella sessilifolia (TUTFWB17 and TUTFWB31) and three (TUTPP4, TUTPP8 and TUTPP10) from P. Pallens could nodulate their original host, due to the unavailability of Wiborgia sericea seeds and the very poor germination of Wiborgia obcordata seeds, authentication of the isolates with their original hosts was not possible. Cowpea was tested as host plant for all 35 isolates, and 86\% of the isolates effectively nodulated cowpea (Table S3).

Salinity tolerance. The rhizobial isolates differed in their response to sodium chloride concentrations. All the 35 isolates could grow in medium supplemented with $0.01 \% \mathrm{NaCl}$ (control) as well as $0.5 \%$ and $1 \% \mathrm{NaCl} .66 \%$ 
and $25 \%$ of isolates tolerated 2 and $3 \% \mathrm{NaCl}$ concentrations, respectively (Table S2). Isolates TUTPP1, TUTPP4 and TUTPP5 from $P$. pallens were susceptible to $2 \% \mathrm{NaCl}$, while isolates TUTFWB17 and TUTFWB31 from $W$. sessilifolia, TUTPP1, TUTPP4, TUTPP5 and TUTPP10 from $P$. pallens, TUTGWO1, TUTGWO3, TUTGWO5, TUTGWO6 TUTGWO7 and TUTGWO12 from $W$. obcordata could tolerate up to $2 \% \mathrm{NaCl}$, susceptible at $3 \%$ $\mathrm{NaCl}$ (Table S2). All isolates from Wiborgia sericea, tolerated up to $3 \% \mathrm{NaCl}$ concentration.

Acidity tolerance. The rhizobial isolates differed in their response to varying $\mathrm{pH}$ levels. All the isolates tested grew in YMA medium pH 7 (control), while, 51\% grew well at pH 5 (Table S2). In contrast, isolates TUTGWO9, TUTGWO11 and TUTGWO15 from Wiborgia obcordata grew at alkaline pH 9-10 (Table S2), while $14 \%$ of the isolates tolerated a wide range of $\mathrm{pH}$ conditions ranging from $\mathrm{pH} 5$ to $\mathrm{pH} 9$, and these included isolates TUTPP3 from Polhillia pallens, TUTGWS2 and TUTGWS3 from Wiborgia sericea, TUTGWO12 and TUTGWO13 from Wiborgiella obcordata.

Screening for phosphate-solubilizing bacteria (PSB). Phosphate-solubilizing bacteria are characteristically identified by the formation of a clear halo around their colonies due to phosphate solubilization on double agar-layered plates. Out of the 35 isolates tested, 34 were able to solubilize tri-calcium phosphate, though the phosphate-solubilizing ability differed as measured by the phosphate-solubilizing index (PSI) (Table S2). Isolate TUTFWB17 from Wiborgiella sessilifolia recorded the largest PSI index (5.0) while isolates TUTGWO9 and TUTGWO11 from Wiborgia obcordata showed the least Index (Table S2). Isolate TUTGWO1 from W. obcordata was incapable of solubilizing $\mathrm{P}$.

Indole acetic acid production. The isolates showed marked differences in their ability to produce IAA in tryptophan supplemented YMB media. Of the 35 isolates tested, $31 \%$ (11 isolates) produced a detectable amount of IAA, which ranged from $0.51 \mu \mathrm{g} \mathrm{ml}^{-1}$ by TUTGWO14 from $W$. obcordata to $51.23 \mu \mathrm{g} \mathrm{ml}^{-1}$ by TUTPP5 from P. pallens (Table S2).

Intrinsic antibiotic resistance. A number of isolates were tolerant to a wide range of antibiotics tested, namely streptomycin, kanamycin, chloramphenicol, ampicillin and neomycin (Table S2). The results showed that 31,3 and $3 \%$ of the 35 test isolates tolerated 50,100 and $200 \mu \mathrm{g} \mathrm{ml}^{-1}$ streptomycin respectively. Isolate TUTPP9 from $P$. pallens was tolerant to $200 \mu \mathrm{g} \mathrm{ml}^{-1}$ streptomycin. However, all the isolates from Wiborgia obcordata were susceptible to streptomycin even at its lowest concentration of $50 \mu \mathrm{g} \mathrm{ml} \mathrm{g}^{-1}$. For kanamycin, $89 \%$ of the test isolates were tolerant to $25 \mu \mathrm{g} \mathrm{ml}^{-1}$, and $11 \%$ susceptible. Only 63 and $29 \%$ at 50 and $75 \mu \mathrm{g} \mathrm{mL} \mathrm{L}^{-1}$, respectively were tolerant to those concentrations of kanamycin. The results also showed that 83,80 and $63 \%$ of the 35 test isolates were tolerant to 25,50 and $75 \mu \mathrm{g} \mathrm{m}^{-1}$ chloramphenicol, respectively. All isolates from Wiborgia sericea, were tolerant to $75 \mu \mathrm{g} \mathrm{ml}^{-1}$, while isolates TUTFWB31 from $W$. sessilifolia, TUTPP5 and TUTPP10 from $P$. pallens, TUTGWO1, TUTGWO2, TUTGWO3 and TUTGWO7 from W. obcordata, were susceptible to $25 \mu \mathrm{g} \mathrm{ml} \mathrm{m}^{-1}$ chloramphenicol. Moreover, a total of 29, 40 and 54\% of the test isolates could not tolerate ampicillin at 25, 50 and $75 \mu \mathrm{g} \mathrm{ml}^{-1}$ concentrations respectively. All W. sericea isolates were tolerant to $75 \mu \mathrm{g} \mathrm{ml}^{-1}$ ampicillin except for isolates TUTGWS3 which was susceptible to $75 \mu \mathrm{g} \mathrm{mL} \mathrm{m}^{-1}$. The majority of $W$ obcordata $(75 \%)$ isolates were susceptible to $75 \mu \mathrm{g} \mathrm{mL}-1$ ampicillin. However, all test isolates (100\%) were resistant to 1 and $5 \mu \mathrm{g} \mathrm{mL} \mathrm{m}^{-1}$ concentrations of neomycin, with $43 \%$ being unable to grow at $10 \mu \mathrm{g} \mathrm{mL}-1$ concentration.

ERIC-PCR amplification. PCR amplification of the ERIC region of the genomic DNA from each isolate yielded distinctive banding patterns. The dendrogram generated from the DNA fingerprints placed the 35 isolates into two major clusters (Fig. 1). Cluster I consisted of 23 isolates obtained from all the host plants with a similarity coefficient of 0.10 . Isolates TUTGWO10, TUTGWO13 and TUTGWO14 from W. obcordata showed the highest similarity coefficients of 1.00 in Cluster I. Twelve mixed isolates from all host plants were grouped in Cluster II. (Fig. 1).

Phylogenetic analysis of the 16S-rRNA gene. The maximum likelihood phylogeny of the 16S-rRNA gene revealed very close sequence similarities of test isolates to the genus Mesorhizobium.

Such that, isolates from Wiborgia obcordata, Wiborgia sericea and Polhillia pallens showed close relationship with Mesorhizobium spp. In cluster I, isolate TUTPP2 from P. pallens was closely related to with M. erdmanii strains and shared $99.4 \%$ sequence identity, while P. pallens isolates TUTPP4, TUTPP5 and TUTPP10 shared 99.1\% sequence identity with $M$. sangaii group as their closest relative in Cluster II. Isolates TUTGWO7, TUTGWO6, TUTGWO14 and TUTGWO2 from W. obcordata and TUTGWS2 from W. sericea revealed 95.0 to $100 \%$ sequence identity with $M$. australicum as the closest relative in Cluster III. W. sessilifolia isolate TUTFWB31 aligned closely with $P$. pallens isolates and together had M. sangaii as the closest relative with $100 \%$ sequence identity in Cluster II (Fig. 2).

Sequence and phylogenetic analyses of housekeeping genes ( $a t p D, g l n l l$ and $g y r B)$. In addition to $16 \mathrm{~S}$ rRNA, three conserved housekeeping genes ( $a t p D$, glnII and gyrB) were selected for phylogenetic analysis. Based on BLASTn, the isolates were placed within the Rhizobium and Mesorhizobium groups. For a clear view of the isolate groupings with reference type strains, separate phylogenies of Rhizobium and Mesorhizobium were constructed (Figs. 3, 4, 5,6). Due to incompatibility of the primer pairs some isolates did not constantly appear in all phylogenies. Isolates from Wiborgia obcordata, P. pallens, W. sericea, and Wiborgiella sessilifolia occupied space in the Mesorhizobium trees with some discrepancies. 


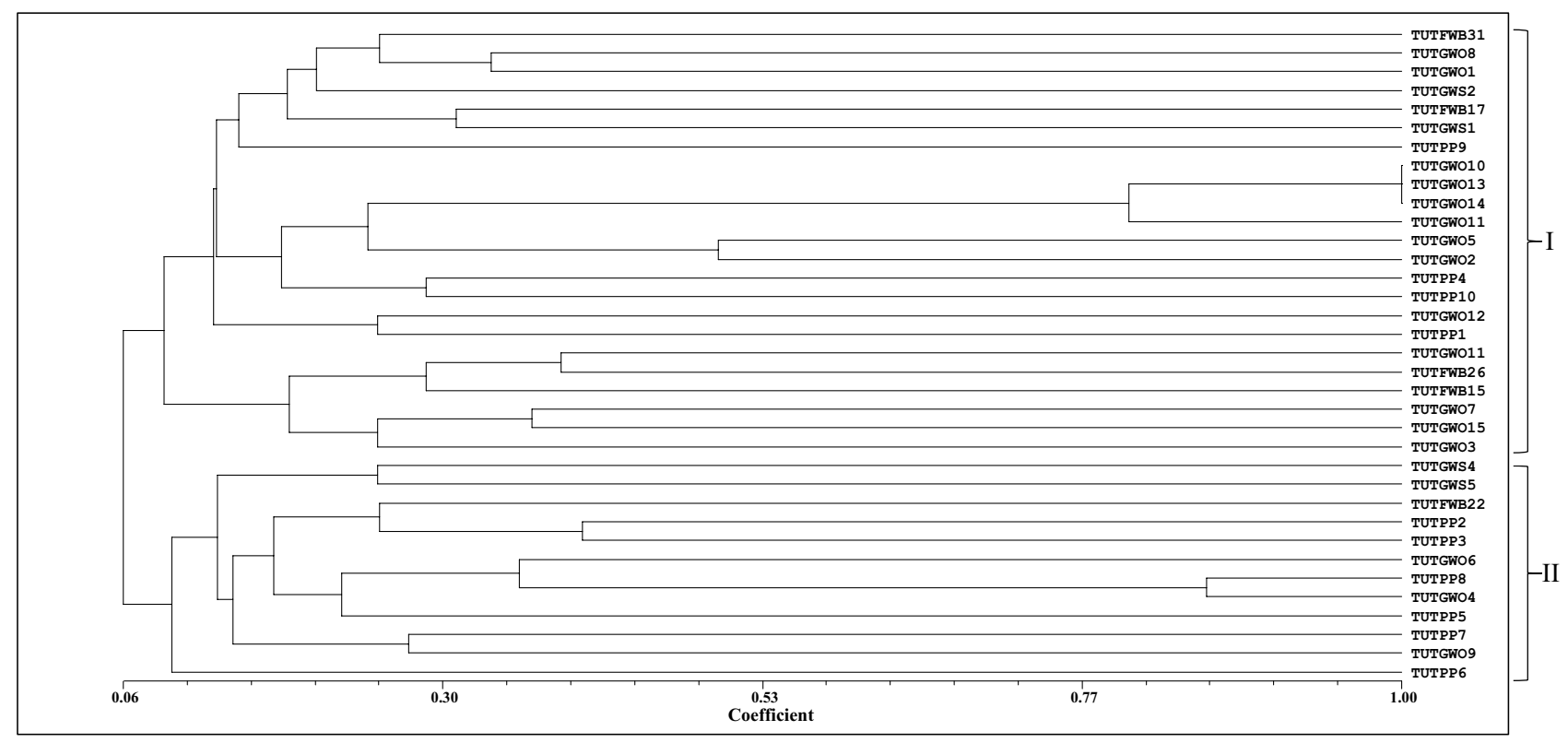

Figure 1. Dendrogram generated from ERIC-PCR fingerprint of 35 test isolates obtained from Wiborgia sericea, Wiborgia obcordata, Wiborgiella sessilifolia and Polhillia pallens.

For example, isolates TUTGWO5, TUTGWO6, TUTGWO7, TUTGWO11 and TUTGWO14 from Wiborgia obcordata were aligned with $M$. australicum as the closest relative with sequence identity ranging from 97.8 (TUTGWO6) to $99.5 \%$ (TUTGWO11) in the atpD phylogram (Fig. 3), 98.1 (TUTGWO7) to 99.6\% (TUTGWO5, TUTGWO6 and TUTGWO11 in the glnII phylogeny (Fig. 4), as well as 95.1 (TUTGWO11) to 95.9\% (TUTWGO6, TUTGWO7 and TUTGWO14) in the gyrB tree (Fig. 6). Surprisingly, some isolates from W. obcordata aligned with Rhizobium in the glnII phylogeny. Isolates TUTGWO8 and TUTGWO9 aligned with R. esperanzae and respectively shared 93.2 and $97.8 \%$ sequence identity, TUTGWO1 aligned with $R$. skierniewicense and shared $90.4 \%$ sequence identity, while isolate TUTGWO2 aligned closely with $R$. leucaenae and shared $97.0 \%$ sequence identity in Clusters III, II and I respectively (Fig. 5).

Similarly, isolates from Wiborgiella sessilifolia which aligned with M. australicum appeared to be closest relative to isolate TUTFWB31 in the $a t p D, g \ln I I$ and $g y r B$ phylogenies with sequence identity of $72.8,94.2$ and 92.7\% respectively. Also, isolates TUTFWB26 and TUTFWB22 had 99.5 and $93.9 \%$ sequence identity with $M$. australicum as the closest relative in the atpD and $g y r B$ phylogenies, respectively (Figs. 3, 6). Interestingly, $g \ln I I$ sequences of isolate TUTFWB15 and TUTFWB26 aligned with Rhizobium spp. and recorded 99.5 and $83.4 \%$ sequence identity respectively with $R$. leucaenae as their closest relative (Fig. 5).

Isolates from Polhillia pallens aligned with Mesorhizobium in the atpD and $g \ln I I$ phylogenies. As found with W. obcordata and W. sessilifolia isolates, some isolates from P. pallens also aligned with Rhizobium in the glnII phylogeny. For instance, isolates TUTPP5 and TUTPP10 aligned together in Cluster I with M. australicum as their closest relative species with sequence identity of 96.0 and $95.15 \%$ in the atpD phylogeny (Fig. 3). Isolates TUTPP4 and TUTPP10 shared a low $84.4 \%$ sequence identity with $M$. australicum as their closest relative in the $g \operatorname{lnII}$ phylogeny (Fig. 4). In contrast, isolate TUTPP9 aligned closely with TUTGWO1 from W. obcordata and shared $97.0 \%$ sequence identity with $R$. skierniewicense as the closest by with relative in Cluster II, while isolates TUTPP2 and TUTPP1 showed sequence identities of 92.2 and $99.5 \%$ respectively with $R$. leucaenae in Cluster I (Fig. 5).

Furthermore, the isolates from Wiborgia sericea aligned with Mesorhizobium and Rhizobium in the gyrB and glnII phylogenies respectively. With the Rhizobium phylogenies, isolates TUTGWS1, TUTGWS4 and TUTGWS5 were identical and had R. leucaenae as a close relative with $99.5 \%$ sequence identity in the glnII phylogeny (Fig. 5). Moreover, isolate TUTGWS2 had R. esperanzae as a closer relative species and together they shared $86.6 \%$ sequence identity in cluster III of the glnII phylogeny (Fig. 5). However, the sequences of isolates TUTGWS2 and TUTGWS4 aligned with $M$. australicum as their closest relative with 93.0 and $90.7 \%$ sequence identity respectively in the gyrB phylogeny (Fig. 6).

Isolates' phylogenetic position based on nifH and nodC genes. Phylogenetic analyses of nifH and nodC genes placed the test isolates closer to the Rhizobium and Mesorhizobium genera in various clusters, similar to the housekeeping gene phylograms (Figs. 7, 8), although some sequence inconsistencies between the phylogenies were observed. Wiborgia obcordata isolates occupied space mainly in the Mesorhizobium phylogeny, though some were found with Rhizobium. Isolates TUTGWO5 aligned closely with some P. pallens isolates and had $92.8 \%$ sequence identity with $M$. chacoence as the closest relative in Cluster II, while isolates TUTGWO6, TUTGWO14, TUTGWO7, TUTGWO11, TUTGWO2 and TUTGWO3 form W. obcordata assembled together in Cluster I and shared a low $90.5 \%$ sequence identity with $M$. chacoense as their closest relative in the nif $H$ phylogeny (Fig. 7). Similarly, in the nodC phylogeny, W. obcordata isolates TUTGWO13, TUTGWO5, TUTGWO1, 


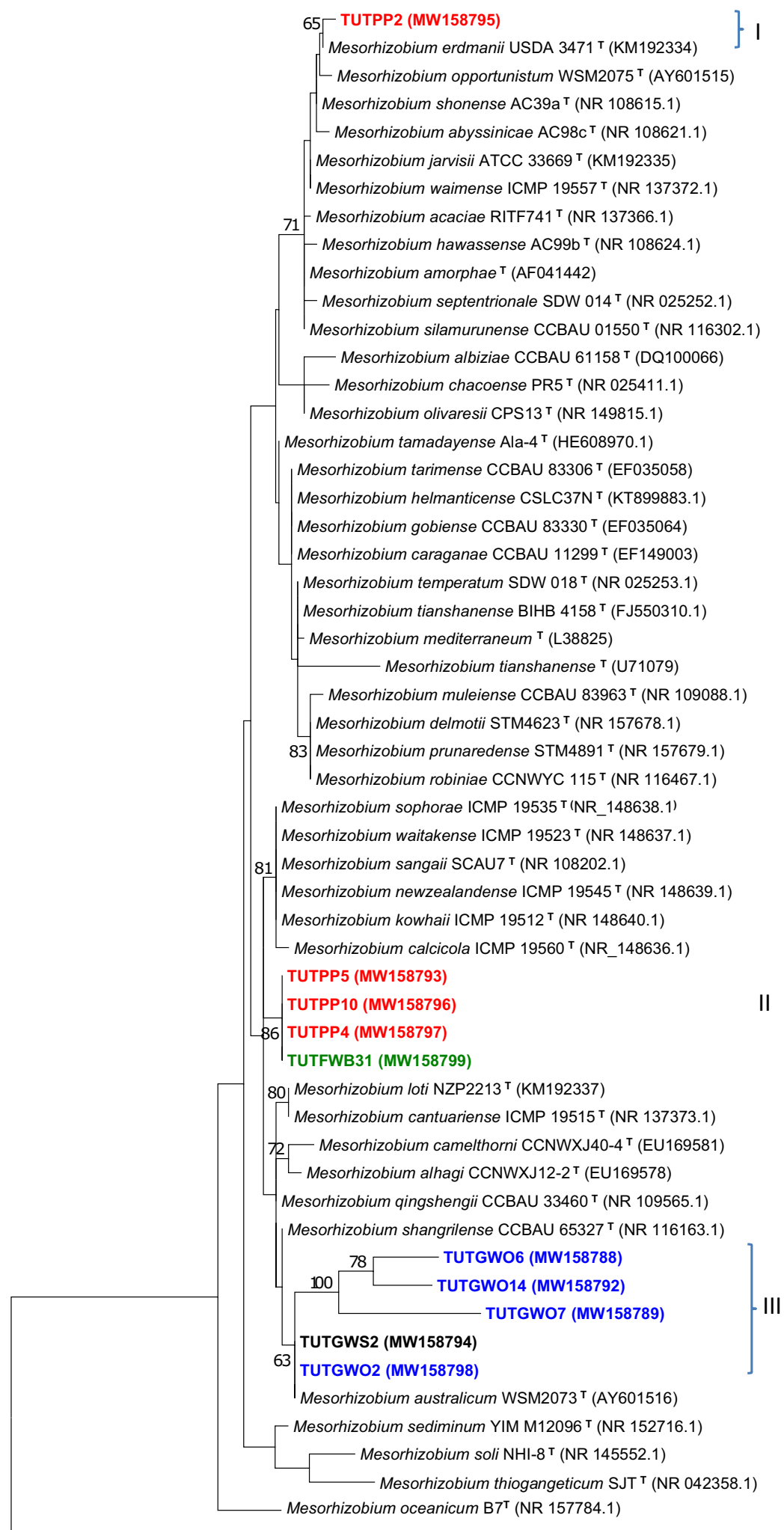

Burkholderia cepacia YBS18 T (MT756628.1)

0.02

Figure 2. The maximum likelihood phylogenetic relationships of root nodule rhizobial isolates isolated from Polhillia pallens (red), Wiborgia obcordata (blue), Wiborgia sericea (black) and Wiborgiella sessilifolia (green), based on Mesorhizobium-16S rRNA sequence analysis. Test isolates are shown in bold. The significance of each branch is indicated by a bootstrap value $=>50$ for each node (1000 replicates). The scale bar represents the number of changes per nucleotide position. 


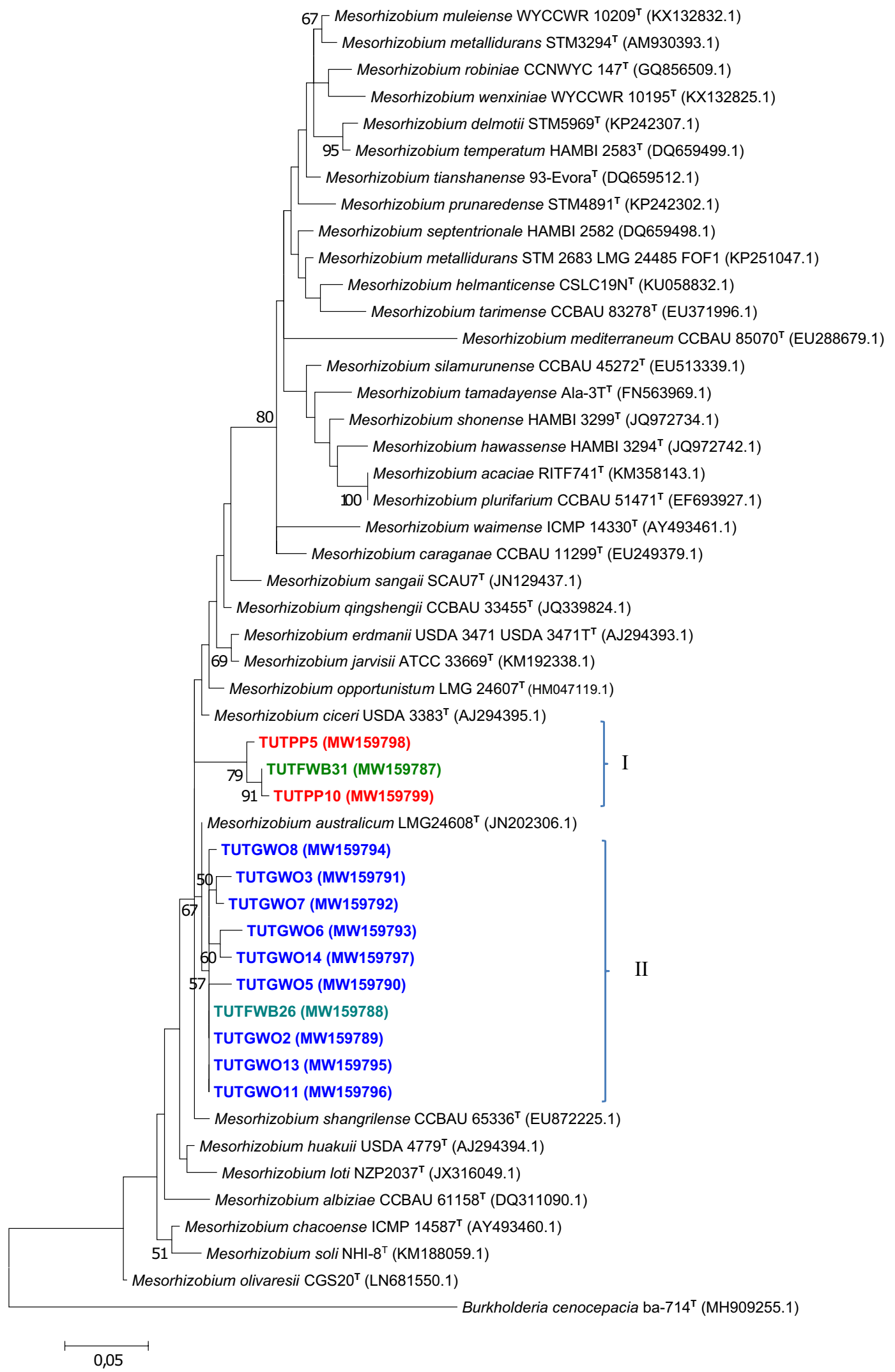

Figure 3. The maximum likelihood phylogenetic relationships of root nodule rhizobial isolates associated with Polhillia pallens (red), Wiborgia obcordata (blue) and Wiborgiella sessilifolia (green), based on MesorhizobiumatpD sequence analysis. Test isolates are shown in bold. The significance of each branch is indicated by a bootstrap value $=>50$ for each node (1000 replicates). The scale bar represents the number of changes per nucleotide position. 


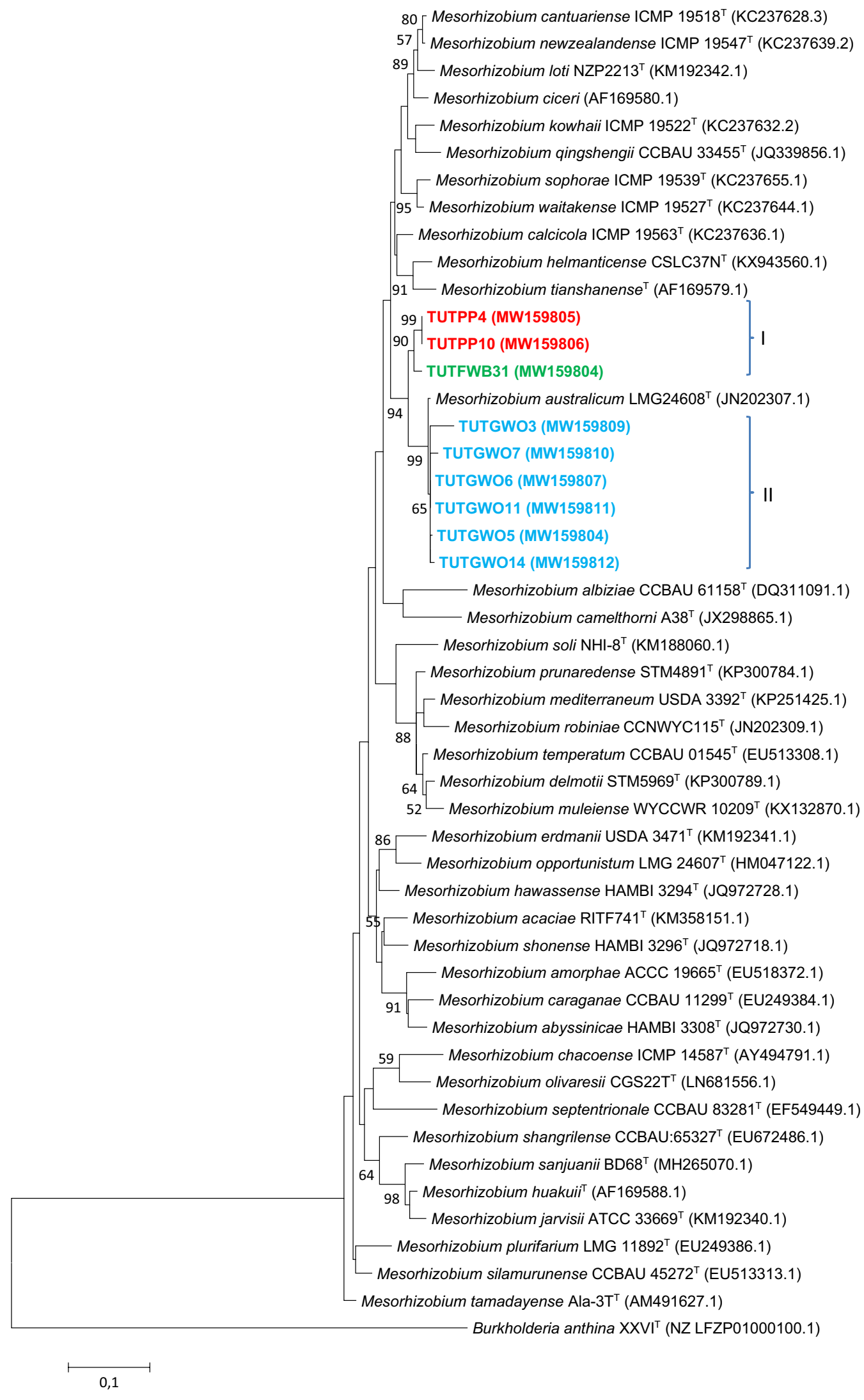

Figure 4. The maximum likelihood phylogenetic relationships of root nodule rhizobial isolates associated with Polhillia pallens (red), Wiborgia obcordata (blue), and Wiborgiella sessilifolia (green), based on MesorhizobiumglnII sequence analysis. Test isolates are shown in bold. The significance of each branch is indicated by a bootstrap value $=>50$ for each node (1000 replicates). The scale bar represents the number of changes per nucleotide position. 


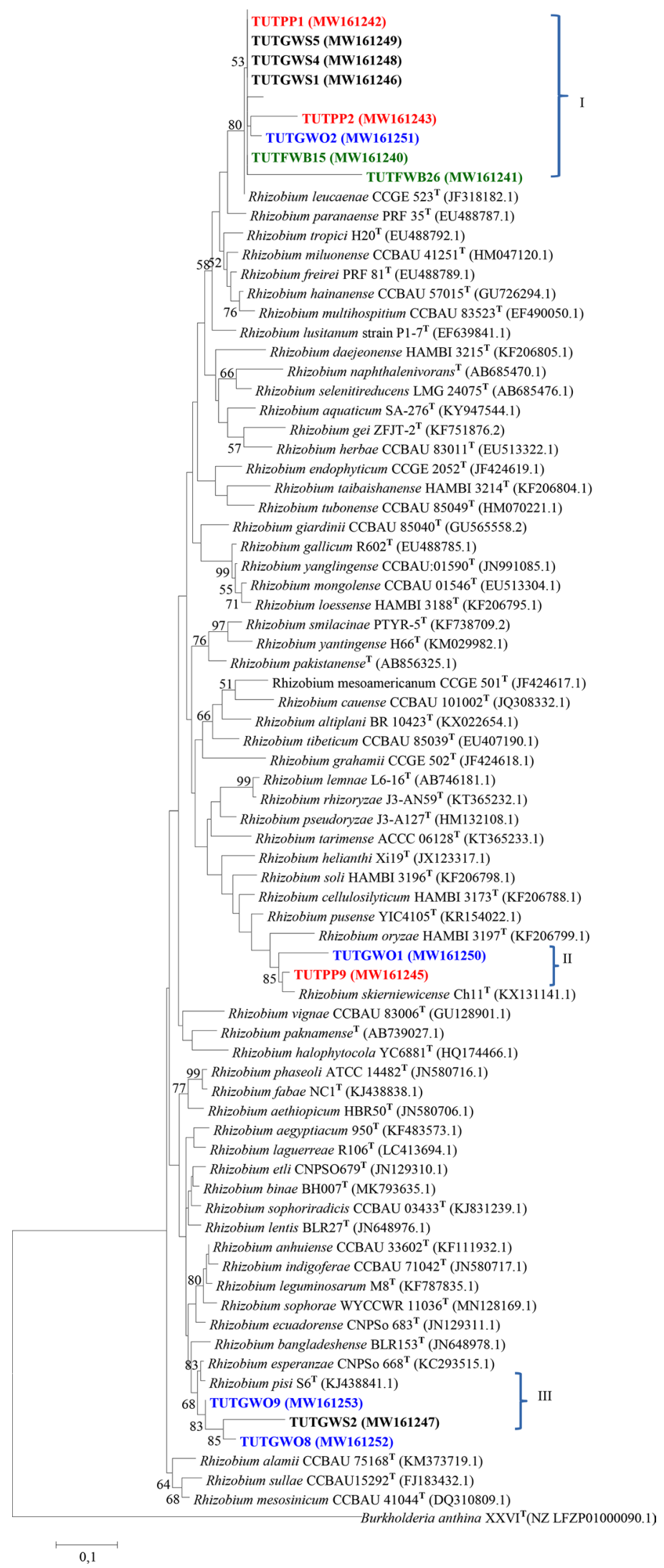

Figure 5. The maximum likelihood phylogenetic relationships of root nodule rhizobial isolates associated with Polhillia pallens (red), Wiborgia obcordata (blue), Wiborgia sericea (black), and Wiborgiella sessilifolia (green), based on Rhizobium-glnII sequence analysis. Test isolates are shown in bold. The significance of each branch is indicated by a bootstrap value $=>50$ for each node ( 1000 replicates). The scale bar represents the number of changes per nucleotide position. 


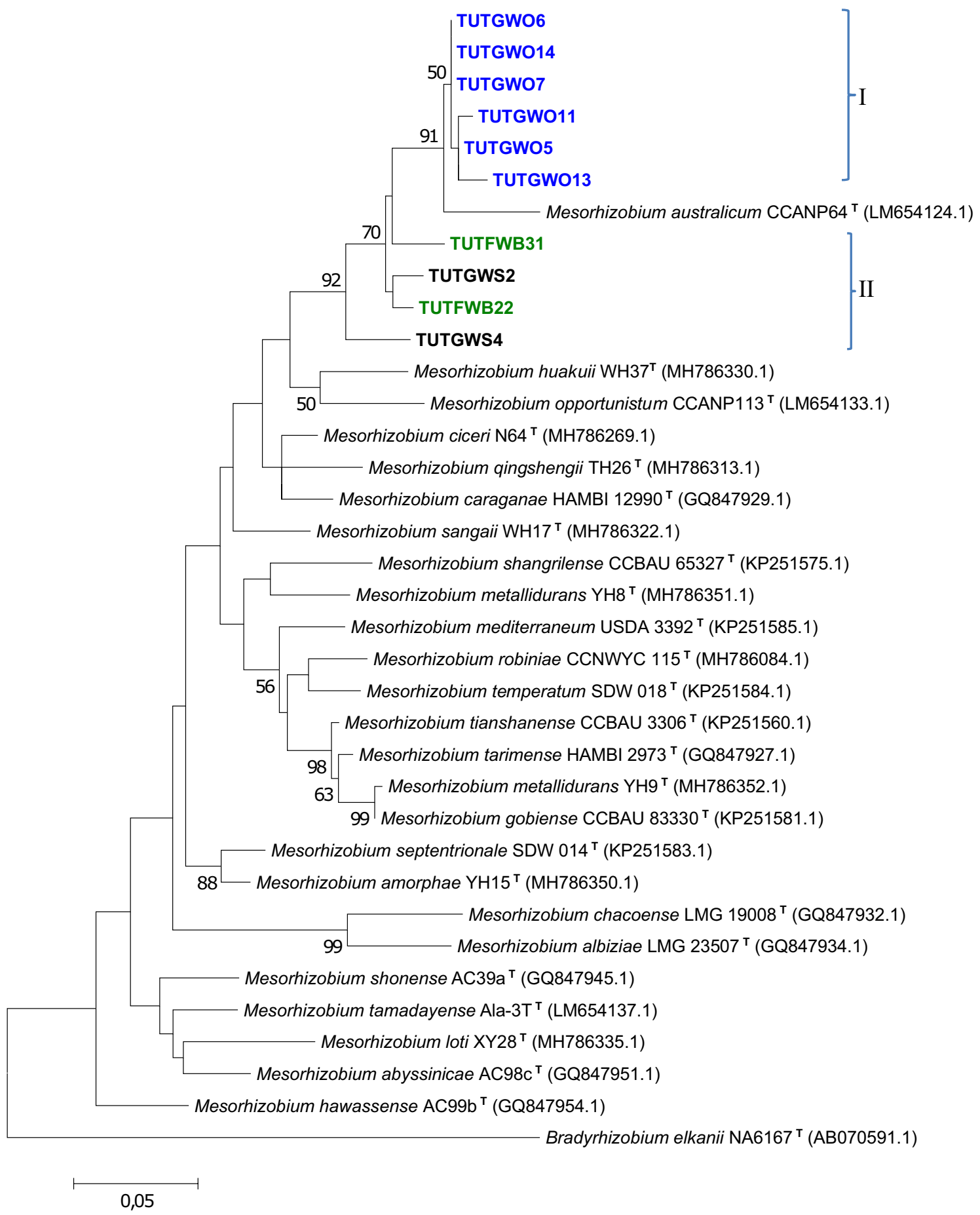

Figure 6. The maximum likelihood phylogenetic relationships of root nodule rhizobial isolates from Wiborgia obcordata (blue), Wiborgia sericea (blue) and Wiborgiella sessilifolia (green) based on Mesorhizobium-gyrB sequence analysis. Test isolates are shown in bold. The significance of each branch is indicated by a bootstrap value $=>50$ for each node (1000 replicates). The scale bar represents the number of changes per nucleotide position.

TUTGWO9, TUTGWO3 and TUTGWO11 aligned together and had a low relationship with the Mesorhizobium reference type strains as they shared between 82.5 and $85.8 \%$ sequence identity with $M$. chacoense, their closest relative in Cluster II (Fig. 8). In contrast to the results from the $16 \mathrm{~S}$ rRNA, atpD, glnII, and gyrB phylogenies, isolates TUTGWO14 and TUTGWO9 aligned with Rhizobium in the nodC and nifH phylogenies respectively, where they shared $99.7 \%$ sequence identity with $R$. tropici as the closest relatives (data not shown).

But, similar to the results obtained from the 16S rRNA and housekeeping phylogenies, isolate TUTFWB31 from W. obcordata aligned with Mesorhizobium in the nodC phylogeny and shared $82.2 \%$ sequence identity with 


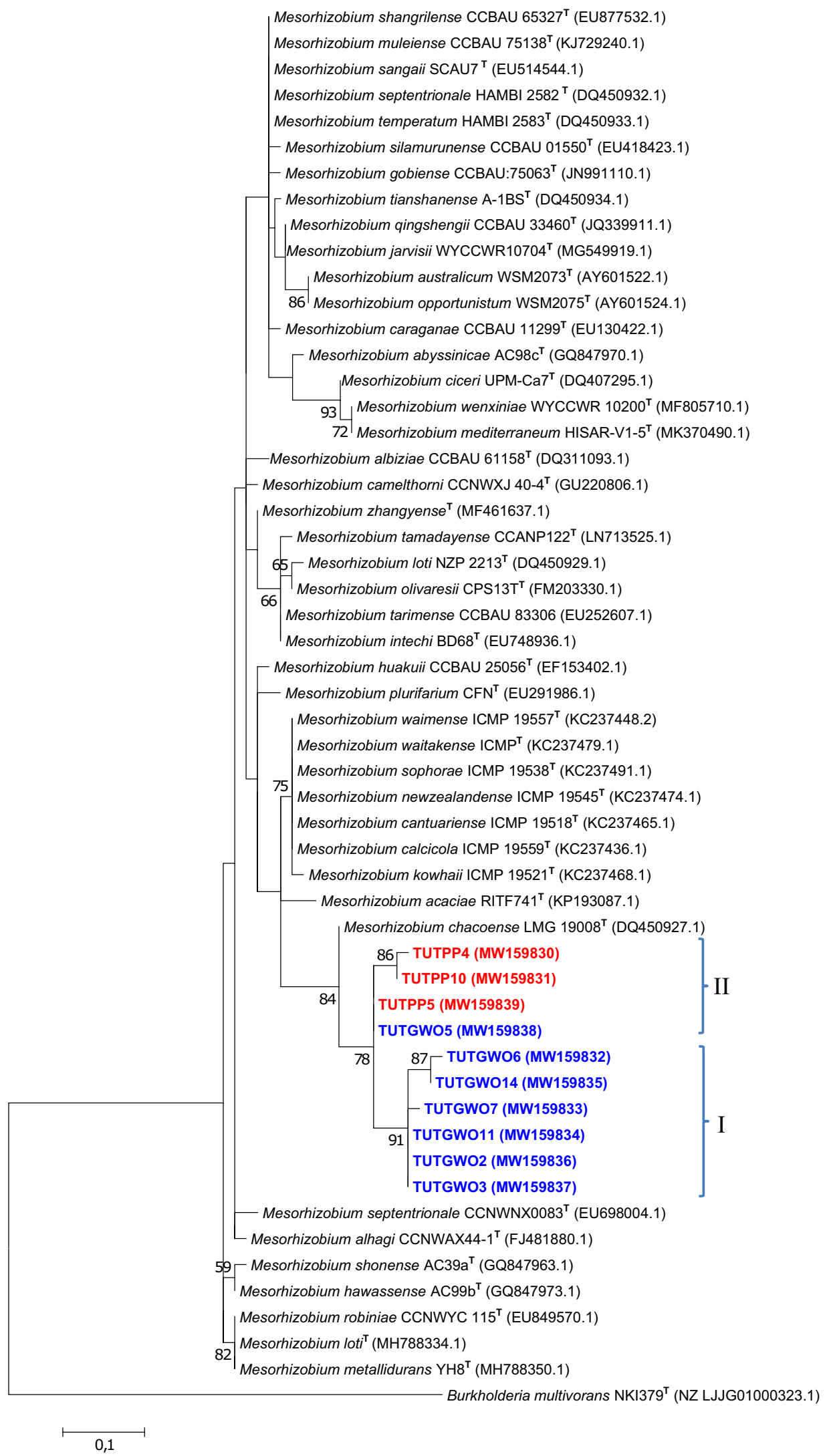

Figure 7. The maximum likelihood phylogenetic relationships of root nodule rhizobial isolates obtained from Polhillia pallens (red) and Wiborgia obcordata (blue) based on Mesorhizobium-nifH sequence analysis. Test isolates are shown in bold. The significance of each branch is indicated by a bootstrap value $=>50$ for each node (1000 replicates). The scale bar represents the number of changes per nucleotide position. 


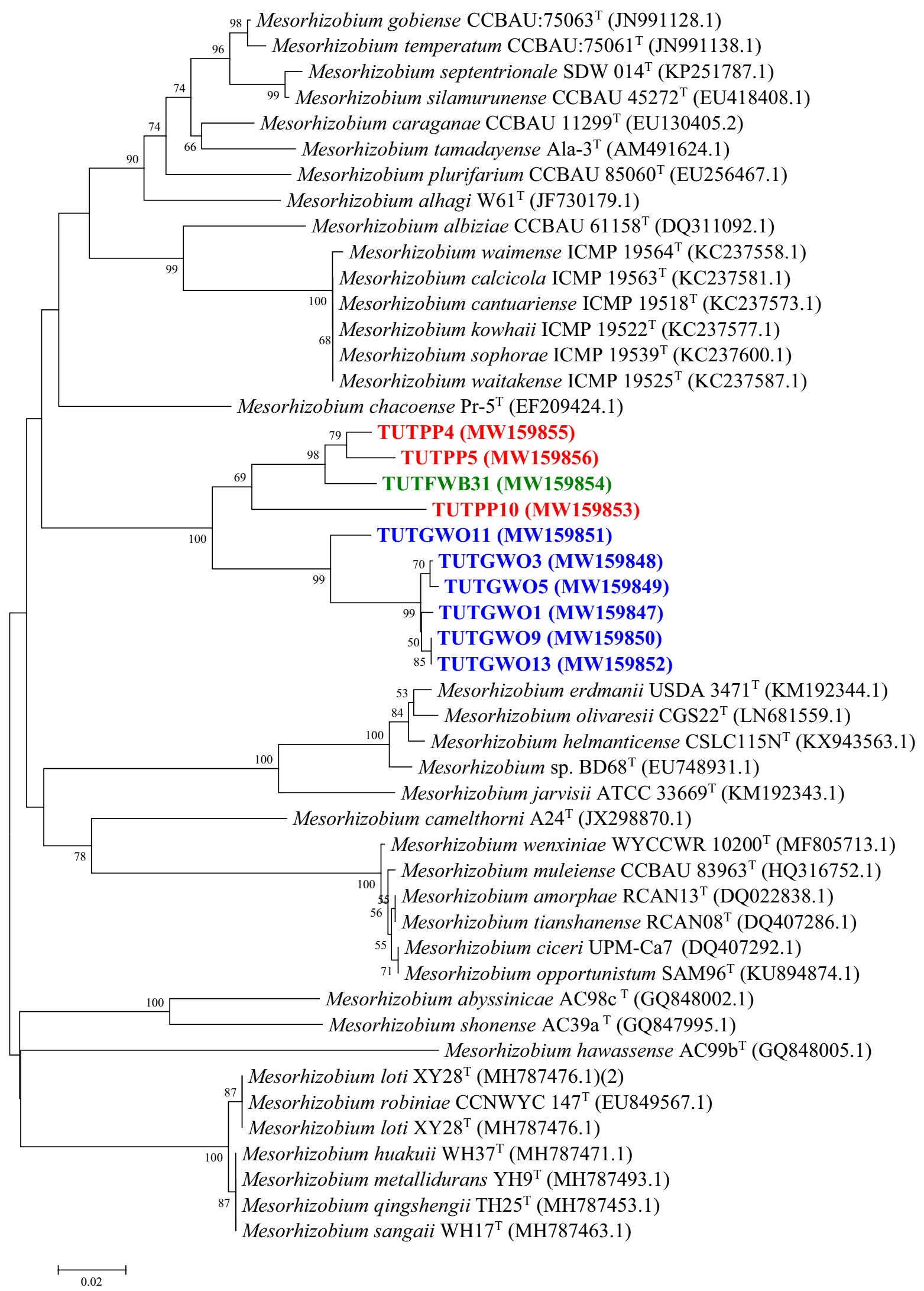

Figure 8. The maximum likelihood phylogenetic relationships of root nodule rhizobial isolate obtained from Polhillia pallens (red), Wiborgia obcordata (blue) and Wiborgiella sessilifolia (green) based on MesorhizobiumnodC sequence analysis. Test isolates are shown in bold. The significance of each branch is indicated by a bootstrap value $=>50$ for each node (1000 replicates). The scale bar represents the number of changes per nucleotide position. 
M. chacoense (Fig. 8). Similarly, Polhillia pallens isolates aligned with Mesorhizobium, and isolates TUTPP4 and TUTPP5 which aligned together in the atpD and glnII phylogenies had M. chacoense (Figs. 3, 4) as their closest relative and shared 90.4 and $92.8 \%$, as well as 83.4 and $82.5 \%$ sequence identity the nifH and nodC phylogenies respectively (Figs. 7,8 ).

\section{Discussion}

Ecological adaptation of native rhizobia to the Cape fynbos. The $\mathrm{N}_{2}$-fixing effectiveness of rhizobia is important for their ability to contribute $\mathrm{N}$ to cropping soil systems and/or the natural environment. However, this can be compromised by various biotic and abiotic factors. Thus, their adaptation to various stress factors is crucial for their survival in the rhizosphere ${ }^{35,36}$. In this study, 35 native rhizobial isolates from the Cape fynbos were tested for their tolerance to different levels of salinity, acidity and antibiotics commonly produced by antagonistic soil-borne microbes. The results revealed strong variations in their tolerance to these environmental factors. The Cape fynbos is generally characterized by sandy acidic soils. The rhizosphere soils from our study sites (except Bredasdorp) were quiet acidic ( $\mathrm{pH} 4.3$ and 5.5), which implies adaption of these isolates to the low $\mathrm{pH}$ soils of the fynbos ${ }^{37}$. It was therefore not surprising that $51 \%$ of the isolates in this study showed tolerance to low $\mathrm{pH}$ ( $\mathrm{pH}$ 5), a finding consistent with the report for Mesorhizobium ${ }^{38}$ in the Cape fynbos.

It was also important to note that Wiborgiella sessilifolia isolates from the alkaline soils of the Bredasdorp site grew better at neutral and acidic $\mathrm{pH} \mathrm{5}$, suggesting their ability to naturally maintain an intracellular $\mathrm{pH}$ of between 7.2 and 7.5 even with an external unfavourable $\mathrm{pH}^{39-41}$. The $19 \%$ of test isolates that tolerated both acidic and alkaline conditions ( $\mathrm{pH} 5$ to $\mathrm{pH}$ 9) closely mirrored the rhizobia reported to nodulate wild Cajanas cajan at $\mathrm{pH} 3$ and 11 and Acacia species at $\mathrm{pH} 4.8$ and $8.8^{41}$. Although alkalinity is less harmful to the survival of bacteria than acidity, it can lead to unavailability of certain essential minerals such as iron and manganese ${ }^{42,43}$, and thus affect plant growth and rhizobial survival. However, three isolates from Wiborgia obcordata which had M. australicum as their closest relative in the $16 \mathrm{~S}$ rRNA, housekeeping and symbiotic gene analysis, could increase their cell division and grow well under alkaline conditions at pH 9 (TUTGWO11 and TUTGWO15) and $\mathrm{pH} 10$ (TUTGWO9).

Furthermore, $72 \%$ of the 35 test isolates were tolerant to $3 \% \mathrm{NaCl}$ concentrations, a finding consistent with an earlier report that isolates from wild legumes can tolerate high $\mathrm{NaCl}(3.5 \%)$ concentrations ${ }^{44}$. High $\mathrm{pH}$ and salinity are also a feature of deserts, such as the Thar desert of India ${ }^{45-47}$, and low $\mathrm{pH}$ is determinant for rhizobial selection by native legumes in central Brazil ${ }^{48}$. With climate change and the potential for an increase in irrigated crop production, soil salinity is likely to become a problem. Therefore, identifying rhizobial isolates with high salinity tolerance would be a solution for increased grain legume production. Additionally, in this study, $35 \%$ of the isolates could produce IAA at high concentrations, even higher than those reported for Mesorhizobium species $^{49}$. IAA is a common by-product of L-tryptophane metabolism in several microorganisms, including rhizobia $^{50}$, and secretion can promote plant root growth and increase nitrogen fixation via upregulation of the genes involved in carbon transport to $\mathrm{N}_{2}$-fixing bacteroids. Thus, $\mathrm{N}_{2}$-fixing rhizobia native to the sandy nutrientpoor soils of the Cape fynbos would have IAA production as an adaptation to supporting root growth of their homologous host legumes. This argument is re-enforced by the fact that the biosynthesis of IAA has been reported in species of Burkholderia, Rhizobium, Mesorhizobium and Bradyrhizobium in the Cape fynbos ${ }^{16,51}$.

Antibiosis or microbial warfare is common in resource-limited soils such as the low nutrients reported for the Cape fynbos. Under those conditions, soil microbes produce antibiotics that can inhibit cell growth and/or kill susceptible bacteria ${ }^{52,53}$. These antibiotics act by inhibiting protein synthesis and are therefore translational inhibitors to the target microbes. In this study, the antibiotic resistance of rhizobial isolates to streptomycin, kanamycin, ampicillin, chloramphenicol and neomycin was evaluated and found to differ markedly among isolates. About $37 \%$ of the isolates were susceptible to $10 \mu \mathrm{g} \mathrm{ml}^{-1}$ concentration of neomycin, an indication that this antibiotic was the least in limiting bacterial growth. Furthermore, $57 \%$ of the isolates in this study were susceptible to streptomycin, contrary to reports that fast-growing isolates from wild legumes are more tolerant of streptomycin ${ }^{54,55}$. More specifically, 15 isolates from Wiborgia obcordata, which were mostly related to Mesorhizobium australicum in the phylogenies, were susceptible to $25 \mu \mathrm{g} \mathrm{ml}{ }^{-1}$ streptomycin. This indicates some vulnerability in their survival in soils that are rich in this antibiotic through inhibition of protein synthesis and translational errors in bacterial cells ${ }^{56}$.

Phylogenetic analysis of microsymbionts nodulating Polhillia, Wiborgia and Wiborgiella in the Cape fynbos. In this study,similarities in isolate alignments and positions were observed in the $\operatorname{glnII}$, gyrB and $a t p D$ phylogenies. For example, in the Mesorhizobium trees, the four isolates TUTGWO5, TUTGWO6, TUTGWO7 and TUTGWO11 from W. obcordata consistently aligned closer to M. australicum reference strain with sequence identity of up to $99.6 \%$, a clear indication that $W$. obcordata is nodulated by M. australicum strain. Furthermore, isolates from P. pallens (TUTPP4 and TUTPP10), W. sericea (TUTGWS2, TUTGWS4) and W. sessilifolia (TUTFWB31 and TUTFWB22) also showed consistency in their alignment with Mesorhizobium reference type strains, with low sequence similarity values $(\leq 97 \%)$, possibly suggesting novel species within Mesorhizobium genus. These results support the reports by Lemaire et $\mathrm{al}^{15}$ and Dludlu et al. ${ }^{17}$, that Mesorhizobium is a common and underestimated nodulator of most legumes in the Cape region, capable of competing effectively with Burkholderia. Further evidence is provided by earlier studies which reported Mesorhizobium species to be compatible with a variety of shrub legumes endemic to fynbos region ${ }^{15,57-59}$.

Some isolates in this study showed incongruency in phylogenies. For example, the phylogenetic analyses of $g \ln I$ for isolates TUTGWS1, TUTGWS2, TUTGWS4, TUTGWS5 from W. sericea, TUTGWO9, TUTGWO8, TUTGWO1, TUTGWO2 from $W$. obcordata and TUTPP9, TUTPP1 and TUTPP2 from P. pallens, as well as isolates TUTFWB26 and TUTFWB15 from W. sessilifolia, suggest that this gene was probably transferred from 
Mesorhizobium to Rhizobium as it showed incongruency with $16 \mathrm{~S}$ rRNA, gyrB, atpD, nodC and nifH phylogenies. Our results therefore agree with reports from Lemaire et al. ${ }^{60}$ who revealed events of horizontal gene transfer between Rhizobium and Mesorhizobium genera in the Cape fynbos region. Furthermore, our results supports the suggestion by Gogarten et al. ${ }^{61}$ who reported that the evidence for potential gene transfer events generally fall into two classes: (1) identification of genes with an unduly high level of similarity to genes found in otherwise unrelated taxa, and (2) genes whose phylogenetic relationships are not congruent with the relationships inferred from other genes in their respective genomes. Reports from Andrew et al. ${ }^{62}$ confirms HGT as a common and unrestricted process which can happen within and between bacterial genera. The disagreement of $g \ln I I$ with $16 \mathrm{~S}$ rRNA phylogeny in this study was also reported by Turner and Young ${ }^{63}$. Phylogenetic analysis of the glutamine synthase gene of rhizobia can also provide strong evidence for horizontal or lateral gene transfer between different genera of rhizobia ${ }^{63}$. Because of possible horizontal gene transfer (or recombination) and variable mutations, single gene-based phylogenetic trees do not always reflect organismal phylogeny ${ }^{64}$.

The identification of Rhizobium glnII gene in isolates TUTGWS2, TUTGWS4, TUTGWS1, TUTGWS5, TUTGWO9, TUTGWO8, TUTGWO1, TUTGWO2, TUTPP9, TUTPP1, TUTPP2, TUTFWB26 and TUTFWB15 strongly supports the view that horizontal transfer of this gene occurred in fynbos soil. Some studies have reported that wild species of Phaseolus such as Phaseolus parvulus, and Phaseolus pauciflorus are nodulated by Bradyrhizobium species ${ }^{65,66}$. A few years ago, Bradyrhizobium paxllaeri and Bradyrhizobium icense were identified in Peru as novel bradyrhizobial species from root nodules of Phaseolus lunatus ${ }^{67}$. Even in Angola within Sub-Saharan Africa, bradyrhizobia were also isolated from common bean nodules ${ }^{68}$.

Isolate TUTGWO14 from W. obcordata grouped with Rhizobium in the nodC phylogeny, but with Mesorhizobium australicum in the $16 \mathrm{~S} \mathrm{rRNA}$, atpD, glnII and gyrB phylogenies. This again suggests a transfer of symbiotic nodC gene from Mesorhizobium to Rhizobium, and thus mirrored the previous reports of the transfer of symbiotic genes between different groups of bacterial species ${ }^{65,69-72}$. Incongruency between the phylogenies of symbiotic (nod and nif) genes and those of chromosomal genes have been reported in a number of studies on rhizobia and has been confirmed as an indication of horizontal inheritance of the symbiosis genes ${ }^{73-75}$. Furthermore, a previous report from the Cape fynbos region has indicated that species within the Crotalarieae are capable of horizontal transfer of symbiosis genes between different genera of rhizobia ${ }^{17}$. Another study indicated has suggested that Sphaerophysa salsula isolates identified as Rhizobium using 16S rRNA gene sequences showed similar nifH sequences to those of the Mesorhizobium isolates, while a Bradyrhizobium isolate (16S rRNA) from Caragana intermedia had similar nodC sequence to the Mesorhizobium isolates ${ }^{76}$.

In this study, the phylogenetic incongruency found between $g \ln I I$ and the $16 \mathrm{~S}$ rRNA, gyrB, atpD, nodC and nifH trees of our isolates indicates their genome plasticity and the lack of clarity in species boundaries, which together support horizontal gene transfer in the test isolates. Ochman et al. ${ }^{77}$ suggested that inter-specific recombination is responsible for the blurring of species boundaries, while phylogenetic incongruency documents gene transfer-mediated organismal diversification. The transfer of core and symbiotic genes between rhizobial genera adapted to local soil conditions can be the consequences of broad mutualistic relationships between test wild legumes and rhizobial genera.

\section{Conclusion}

The morpho-genetically diverse rhizobia isolated from Polhillia, Wiborgia, and Wiborgiella species from the Cape fynbos region of South Africa were found to tolerate exposure to factors such as acidity, alkalinity, salinity and antibiotics. These isolates also differed in their varying abilities to solubilize P and/or produce IAA, thus suggesting varying ability to promote plant growth. In this study, Mesorhizobium australicum is the microsymbiont nodulating Wiborgia obcordata, while Polhillia pallens, Wiborgia sericea and Wiborgiella sessilifolia are nodulated by some possible novel Mesorhizobium spp.. The genomes arrangement of the test isolates indicate genetic plasticity which suggests the need to evaluate the symbiotic functioning and competitive advantage of these isolates using their homologous host plants.

\section{Data availability}

Data used in this study are available under following accession numbers. 16SrRNA (MW158788-MW158799), atpD (MW159787- MW159799), glnII (MW159804-MW159813), gyrB (MW159814- MW159823); nifH (MW159830-MW159846, MW161258); nodC (MW159847- MW159861).

Received: 28 May 2021; Accepted: 18 November 2021

Published online: 08 December 2021

\section{References}

1. Stirton, C. H. Polhillia, a new genus of papilionoid legumes endemic to South Africa. South African J. Bot. 52, 167-180 (1986).

2. Boatwright, J. S., Tilney, P. M. \& Van Wyk, B.-E. Taxonomy of Wiborgiella (Crotalarieae, Fabaceae), a genus endemic to the greater Cape Region of South Africa. Syst. Bot. 35, 325-340 (2010).

3. Moiloa, N. A., Chimphango, S. B. M. \& Muasya, A. M. A phylogenetic study of the genus Wiborgia (Crotalarieae, Fabaceae). South African J. Bot. 115, 179-193 (2018).

4. Myers, N., Mittermeier, R. A., Mittermeier, C. G., Da Fonseca, G. A. B. \& Kent, J. Biodiversity hotspots for conservation priorities. Nature 403, 853 (2000)

5. Goldblatt, P. \& Manning, J. C. Plant diversity of the Cape region of southern Africa. Ann. Missouri Bot. Gard. 281-302 (2002)

6. Forest, F., Colville, J. F. \& Cowling, R. M. Evolutionary diversity patterns in the Cape flora of South Africa. in Phylogenetic Diversity 167-187 (Springer, 2018).

7. Boatwright, J. S. \& Cupido, C. N. Aspalathus crewiana sp. Nov. (Crotalarieae, Fabaceae) from the Western Cape Province, South Africa. Nord. J. Bot. 29, 513-517 (2011). 
8. Mpai, T., Jaiswal, S. K. \& Dakora, F. D. Accumulation of phosphorus and carbon and the dependency on biological N-2 fixation for nitrogen nutrition in Polhillia, Wiborgia and Wiborgiella species growing in natural stands in cape fynbos, South Africa. SYMBIOSIS (2020).

9. Van Zwieten, L. et al. Enhanced biological N 2 fixation and yield of faba bean (Vicia faba L.) in an acid soil following biochar addition: Dissection of causal mechanisms. Plant Soil 395, 7-20 (2015).

10. Jaiswal, S. K., Naamala, J. \& Dakora, F. D. Nature and mechanisms of aluminium toxicity, tolerance and amelioration in symbiotic legumes and rhizobia. Biol. Fertil. Soils https://doi.org/10.1007/s00374-018-1262-0 (2018).

11. Araújo, S. S. et al. Abiotic stress responses in legumes: Strategies used to cope with environmental challenges. CRC. Crit. Rev. Plant Sci. 34, 237-280 (2015).

12. Etesami, H., Alikhani, H. \& Akbari, A. Evaluation of plant growth hormones production (IAA) ability by Iranian soils rhizobial strains and effects of superior strains application on wheat growth. World Appl. Sci. J. 6, 1576-1584 (2009).

13. Ibny, F. Y. I., Jaiswal, S. K., Mohammed, M. \& Dakora, F. D. Symbiotic effectiveness and ecologically adaptive traits of native rhizobial symbionts of Bambara groundnut (Vigna subterranea L. Verdc.) in Africa and their relationship with phylogeny. Sci. Rep. 9, $1-17$ (2019).

14. Kanu, S. A. \& Dakora, F. D. Symbiotic nitrogen contribution and biodiversity of root-nodule bacteria nodulating Psoralea species in the Cape Fynbos, South Africa. Soil Biol. Biochem. 54, 68-76 (2012).

15. Lemaire, B. et al. Symbiotic diversity, specificity and distribution of rhizobia in native legumes of the Core Cape Subregion (South Africa). FEMS Microbiol. Ecol. 91, 2-17 (2015).

16. Brink, C., Postma, A. \& Jacobs, K. Rhizobial diversity and function in rooibos (Aspalathus linearis) and honeybush (Cyclopia spp.) plants: A review. South African J. Bot. 110, 80-86 (2017).

17. Dludlu, M. N., Chimphango, S. B. M., Walker, G., Stirton, C. H. \& Muasya, A. M. Horizontal gene transfer among rhizobia of the Core Cape Subregion of southern Africa. South African J. Bot. 118, 342-352 (2018).

18. Aliero, B. L. Effects of sulphuric acid, mechanical scarification and wet heat treatments on germination of seeds of African locust bean tree, Parkia biglobosa. African J. Biotechnol. 3, 179-181 (2004).

19. Hematifar, M., Tehranifar, A. \& Abedi, B. Facilitating Seed Germination of Eight Species of Hawthorn (Crataegus spp.) Native of Iran, Using Chemical Scarification and Cold Stratification. Iran. J. Seed Res. 4, 13-22 (2018).

20. Vincent, J. M. A Manual for the Practical Study of Root-Nodule Bacteria: A Manual for the Practical Study of Root-Nodule Bacteria Vol. 15 (Blackwell Scientific, 1970).

21. Unkovich, M. \& Baldock, J. Measurement of asymbiotic N2 fixation in Australian agriculture. Soil Biol. Biochem. 40, 2915-2921 (2008).

22. Somasegaran, P. \& Hoben, H. J. Handbook for Rhizobia: Methods in Legume-Rhizobium Technology (Springer, 2012).

23. Sneath, P. H. A., Sokal, R. R. Numerical taxonomy. The principles and practice of numerical classification. (1973).

24. Rohlf, F. J., Applied Biostatistics, I. \& Exeter Software (Firm). NTSYS-pc: Numerical taxonomy and multivariate analysis system. (Applied Biostatistics, Inc., 2009)

25. Hall, T. BioEdit version 7.0. 0. Distributed by the author, website: www.mbio.ncsu.edu/BioEdit/bioedit.html. (2004).

26. Edgar, R. C. MUSCLE: Multiple sequence alignment with high accuracy and high throughput. Nucleic Acids Res. 32, 1792-1797 (2004).

27. Tamura, K., Stecher, G., Peterson, D., Filipski, A. \& Kumar, S. MEGA6: Molecular evolutionary genetics analysis version 6.0. Mol. Biol. Evol. 30, 2725-2729 (2013).

28. Nei, M. \& Kumar, S. Molecular Evolution and Phylogenetics (Oxford University Press, 2000).

29. Saitou, N. \& Nei, M. The neighbor-joining method : A new method for reconstructing phylogenetic trees. Mol. Biol. Evol. 4, 406-425 (1987).

30. Felsenstein, J. Confidence limits on phylogenies: An approach using the bootstrap. Evolution 39, 783-791 (1985).

31. Morón, B. et al. Low pH changes the profile of nodulation factors produced by Rhizobium tropici CIAT899. Chem. Biol. 12, 1029-1040 (2005).

32. Moroenyane, I., Chimphango, S. B. M., Wang, J., Kim, H. K. \& Adams, J. M. Deterministic assembly processes govern bacterial community structure in the Fynbos, South Africa. Microb. Ecol. 72, 313-323 (2016).

33. Dabo, M., Jaiswal, S. K. \& Dakora, F. D. Phylogenetic evidence of allopatric speciation of bradyrhizobia nodulating cowpea ( Vigna unguiculata L. walp ) in South African and Mozambican soils Department of Crop Sciences, Tshwane University of Technology, Private Bag Chemistry Department. Tshw. FEMS Microbiol. Ecol. 19, 1-14 (2019).

34. Singh, S. K., Jaiswal, S. K., Vaishampayan, A. \& Dhar, B. Physiological behavior and antibiotic response of soybean (Glycine max L.) nodulating rhizobia isolated from Indian soils. African J. Microbiol. Res. 7, 2093-2102 (2013).

35. Hayat, R., Ali, S., Amara, U., Khalid, R. \& Ahmed, I. Soil beneficial bacteria and their role in plant growth promotion: A review. Ann. Microbiol. 60, 579-598 (2010).

36. Berendsen, R. L., Pieterse, C. M. J. \& Bakker, P. A. H. M. The rhizosphere microbiome and plant health. Trends Plant Sci. 17, $478-486$ (2012).

37. Maseko, S. T. \& Dakora, F. D. Rhizosphere acid and alkaline phosphatase activity as a marker of P nutrition in nodulated Cyclopia and Aspalathus species in the Cape fynbos of South Africa. South African J. Bot. 89, 289-295 (2013).

38. Dludlu, M. N., Chimphango, S., Stirton, C. H. \& Muasya, A. M. Differential preference of burkholderia and mesorhizobium to $\mathrm{pH}$ and soil types in the core cape subregion, South Africa. Genes 9, 2 (2017).

39. Graham, P. H. et al. Acid pH tolerance in strains of Rhizobium and Bradyrhizobium, and initial studies on the basis for acid tolerance of Rhizobium tropici UMR1899. Can. J. Microbiol. 40, 198-207 (1994).

40. Fikri-Benbrahim, K., Chraibi, M., Lebrazi, S., Moumni, M. \& Ismaili, M. Phenotypic and Genotypic Diversity and Symbiotic Effectiveness of Rhizobia Isolated from Acacia sp. Grown in Morocco. J. Agric. Sci. Technol. 19, (2017).

41. Moumni, M., Fikri-Benbrahim, K., Ismaili, M., Lebrazi, S. \& Chraibi, M. Phenotypic and G enotypic D iversity and S ymbiotic E ffectiveness of R hizobia I solated from Acacia sp. G rown in Morocco. JKUAT (2018). http://hdl.handle.net/123456789/3738

42. Farissi, M. et al. Growth, nutrients concentrations, and enzymes involved in plants nutrition of alfalfa populations under saline conditions. (2014).

43. Lebrazi, S. \& Benbrahim, K. F. Environmental stress conditions affecting the N2 fixing Rhizobium-legume symbiosis and adaptation mechanisms. African J. Microbiol. Res. 8, 4053-4061 (2014).

44. Bhargava, Y., Murthy, J. S. R., Kumar, T. V. R. \& Rao, M. N. Phenotypic, stress tolerance and plant growth promoting characteristics of rhizobial isolates from selected wild legumes of semiarid region, Tirupati, India. Adv. Microbiol. 6, 1 (2016).

45. Sankhla, I. S. et al. Molecular characterization of nitrogen fixing microsymbionts from root nodules of Vachellia (Acacia) jacquemontii, a native legume from the Thar Desert of India. Plant Soil 410, 21-40 (2017).

46. Rathi, S. et al. Selection of Bradyrhizobium or Ensifer symbionts by the native Indian caesalpinioid legume Chamaecrista pumila depends on soil pH and other edaphic and climatic factors. FEMS Microbiol. Ecol. 94, 1-17 (2018).

47. Choudhary, D., Rai, M. K., Shekhawat, N. S. \& Kataria, V. In vitro propagation of Farsetia macrantha Blatt. I\& Hallb.: An endemic and threatened plant of Indian Thar Desert. Plant Cell, Tissue Organ Cult. 142, 519-526 (2020).

48. de Castro Pires, R. et al. Soil characteristics determine the rhizobia in association with different species of Mimosa in central Brazil. Plant Soil 423, 411-428 (2018). 
49. Verma, J. P., Yadav, J., Tiwari, K. N. \& Kumar, A. Effect of indigenous Mesorhizobium spp. and plant growth promoting rhizobacteria on yields and nutrients uptake of chickpea (Cicer arietinum L.) under sustainable agriculture. Ecol. Eng. 51, 282-286 (2013).

50. Datta, C. \& Basu, P. S. Indole acetic acid production by a Rhizobium species from root nodules of a leguminous shrub, Cajanus cajan. Microbiol. Res. 155, 123-127 (2000).

51. Brink, C. J. Plant Growth-Promoting Properties of Fynbos Rhizobia and Their Diversity (Stellenbosch University, 2018).

52. Naamala, J., Jaiswal, S. K. \& Dakora, F. D. Antibiotics resistance in Rhizobium: Type, process, mechanism and benefit for agriculture. Curr. Microbiol. 72, 804-816 (2016).

53. Baba, T. \& Schneewind, O. Instruments of microbial warfare: Bacteriocin synthesis, toxicity and immunity. Trends Microbiol. 6, 66-71 (1998).

54. Menezes, K. A. S., Nunes, G. F. O. \& Sampaio, A. A. Diversity of new root nodule bacteria from Erythrina velutina Willd., a native legume from the Caatinga dry forest (Northeastern Brazil). Rev Cienc Agrárias 39, 222-233 (2016).

55. Pagano, M. C. Rhizobia associated with neotropical tree Centrolobium tomentosum used in riparian restoration. Plant, Soil Environ. 54, 498-508 (2008).

56. Hong, W., Zeng, J. \& Xie, J. Antibiotic drugs targeting bacterial RNAs. Acta Pharm. Sin. B 4, 258-265 (2014).

57. Elliott, G. N. et al. Nodulation of Cyclopia spp. (Leguminosae, Papilionoideae) by Burkholderia tuberum. Ann. Bot. 100, 1403-1411 (2007).

58. Hassen, A. I., Bopape, F. L., Habig, J. \& Lamprecht, S. C. Nodulation of rooibos (Aspalathus linearis Burm. f.), an indigenous South African legume, by members of both the $\alpha$-proteobacteria and $\beta$-proteobacteria. Biol. Fertil. Soils 48, 295-303 (2012).

59. Gerding, M., O’Hara, G. W., Bräu, L., Nandasena, K. \& Howieson, J. G. Diverse Mesorhizobium spp. with unique nodA nodulating the South African legume species of the genus Lessertia. Plant Soil 358, 385-401 (2012).

60. Lemaire, B. et al. Recombination and horizontal transfer of nodulation and ACC deaminase (acdS) genes within Alpha-and Betaproteobacteria nodulating legumes of the Cape Fynbos biome. FEMS Microbiol. Ecol. 91, (2015).

61. Gogarten, J. P., Doolittle, W. F. \& Lawrence, J. G. Prokaryotic evolution in light of gene transfer. Mol. Biol. Evol. 19, 2226-2238 (2002).

62. Andrews, M. et al. Horizontal transfer of symbiosis genes within and between rhizobial genera: Occurrence and importance. Genes 9, 321 (2018).

63. Turner, S. L. \& Young, J. P. W. The glutamine synthetases of rhizobia : Phylogenetics and evolutionary implications. 17, 309-319 (2000).

64. Gevers, D. et al. Re-evaluating prokaryotic species. Nat. Rev. Microbiol. 3, 733 (2005).

65. Ormeño-Orrillo, E. et al. Phylogenetic evidence of the transfer of nodZ and nolL genes from Bradyrhizobium to other rhizobia. Mol. Phylogenet. Evol. 67, 626-630 (2013).

66. Parker, M. A., Lafay, B., Burdon, J. J. \& Van Berkum, P. Conflicting phylogeographic patterns in rRNA and nifD indicate regionally restricted gene transfer in Bradyrhizobiumaa. Microbiology 148, 2557-2565 (2002).

67. Duran, D. et al. Bradyrhizobium paxllaeri sp. Nov. and Bradyrhizobium icense sp. Nov., nitrogen-fixing rhizobial symbionts of Lima bean (Phaseolus lunatus L.) in Peru. Int. J. Syst. Evol. Microbiol. 64, 2072-2078 (2014).

68. Grönemeyer, J. L., Kulkarni, A., Berkelmann, D., Hurek, T. \& Reinhold-Hurek, B. Identification and characterization of rhizobia indigenous to the Okavango region in Sub-Saharan Africa. Appl. Environ. Microbiol. https://doi.org/10.1128/AEM.02417-14 (2014).

69. Rogel, M. A., Ormeno-Orrillo, E. \& Romero, E. M. Symbiovars in rhizobia reflect bacterial adaptation to legumes. Syst. Appl. Microbiol. 34, 96-104 (2011).

70. Lindstrom, K., Murwira, M., Willems, A. \& Altier, N. The biodiversity of beneficial microbe-host mutualism : The case of rhizobia. Res. Microbiol. 161, 453-463 (2010).

71. Barcellos, F. G., Menna, P., da Silva Batista, J. S. \& Hungria, M. Evidence of horizontal transfer of symbiotic genes from a Bradyrhizobium japonicum inoculant strain to indigenous diazotrophs Sinorhizobium (Ensifer) fredii and Bradyrhizobium elkanii in a Brazilian Savannah soil. Appl. Environ. Microbiol. 73, 2635-2643 (2007).

72. Jourand, P., Mateille, T., Fargette, M. \& Rapior, S. Nematostatic activity of aqueous extracts of West African Crotalaria species. Nematology 6, 765-771 (2004).

73. Chen, W.-M. et al. Legume symbiotic nitrogen fixation by $\beta$-proteobacteria is widespread in nature. J. Bacteriol. 185, 7266-7272 (2003).

74. Aoki, S., Ito, M. \& Iwasaki, W. From $\beta$-to $\alpha$-proteobacteria: The origin and evolution of rhizobial nodulation genes nodIJ. Mol. Biol. Evol. 30, 2494-2508 (2013).

75. Moulin, L., Béna, G., Boivin-Masson, C. \& Stkepkowski, T. Phylogenetic analyses of symbiotic nodulation genes support vertical and lateral gene co-transfer within the Bradyrhizobium genus. Mol. Phylogenet. Evol. 30, 720-732 (2004).

76. Lu, Y. L. et al. Genetic diversity and biogeography of rhizobia associated with Caragana species in three ecological regions of China. Syst. Appl. Microbiol. 32, 351-361 (2009).

77. Ochman, H., Lawrence, J. G. \& Groisman, E. A. Lateral gene transfer and the nature of bacterial innovation. Nature 405, 299-304 (2000).

\section{Acknowledgements}

This study was supported with grants from the National Research Foundation, the Department of Science and Technology, Tshwane University of Technology, and the South African Research Chair in Agrochemurgy and Plant Symbioses. TM is grateful to GreenMatter for providing a partial Scholarship. We thank Mr Matthias Streicher and his family for granting us permission to collect seeds of Polhillia species on their farm, as well as to sample rhizosphere soils for use as bacterial inoculum for plant nodulation in the glasshouse. We are also grateful to the Cape Nature Conservation for granting us a Permit to sample plant material, rhizosphere soils, and where possible, root nodules from Wiborgia and Wiborgiella species from various locations in the Cape fynbos.

\section{Author contributions}

T.M. performed the experiments. T.M. and S.K.J. analysed data and drafted the manuscript. C.N.C. helped in samples collection from fynbos region. F.D.D. edited the manuscript and provided funding for the study.

\section{Competing interests}

The authors declare no competing interests.

\section{Additional information}

Supplementary Information The online version contains supplementary material available at https://doi.org/ 10.1038/s41598-021-02766-2. 
Correspondence and requests for materials should be addressed to S.K.J. or F.D.D.

Reprints and permissions information is available at www.nature.com/reprints.

Publisher's note Springer Nature remains neutral with regard to jurisdictional claims in published maps and institutional affiliations.

(c) (i) Open Access This article is licensed under a Creative Commons Attribution 4.0 International cc) License, which permits use, sharing, adaptation, distribution and reproduction in any medium or format, as long as you give appropriate credit to the original author(s) and the source, provide a link to the Creative Commons licence, and indicate if changes were made. The images or other third party material in this article are included in the article's Creative Commons licence, unless indicated otherwise in a credit line to the material. If material is not included in the article's Creative Commons licence and your intended use is not permitted by statutory regulation or exceeds the permitted use, you will need to obtain permission directly from the copyright holder. To view a copy of this licence, visit http://creativecommons.org/licenses/by/4.0/.

(C) The Author(s) 2021 